\title{
Theoretical Insight into the Spectroscopy and Photochemistry of Isoalloxazine, the Flavin Core Ring
}

\author{
Supporting Information
}

Teresa Climent, Remedios González-Luque, Manuela Merchán, and Luis Serrano-Andrés*

\author{
Instituto de Ciencia Molecular \\ Universitat de València \\ Apartado 22085 \\ ES-46071 Valencia \\ Spain
}

* To whom correspondence should be addressed. E-mail address: Luis.Serrano@uv.es 


\section{Complete reference 39 of the paper:}

Andersson, K.; Barysz, M.; Bernhardsson, A.; Blomberg, M. R. A.; Carissan, Y.; Cooper, D. L.; Cossi, M.; Fülscher, M. P.; Gagliardi, L.; de Graaf, C.; Hess, B.; Hagberg, G.; Karlström, G.; Lindh, R.; Malmqvist, P.-Å.; Nakajima, T.; Neogrády, P.; Olsen, J.; Raab, J.; Roos, B. O.; Ryde, U.; Schimmelpfennig, B.; Schütz, M.; Seijo, L.; Serrano-Andrés, L.; Siegbahn, P. E. M.; Stålring, J.; Thorsteinsson, T.; Veryazov, V.; Widmark, P.-O. MOLCAS, version 6.0; Department of Theoretical Chemistry, Chemical Centre, University of Lund: Lund, Sweden, 2004. 
Table SI1. Cartesian coordinates $(\AA)$ for the relevant structures of the isoalloxazine ring.

$\mathrm{X}$

\section{$\mathrm{y}$}

Z

(gs) $\min$

$\begin{array}{lrrr}\mathrm{N} & 2.316339 & 0.165622 & 0.000000 \\ \mathrm{~N} & 1.745279 & 2.490236 & 0.000000 \\ \mathrm{~N} & 0.650881 & -1.397571 & 0.000000 \\ \mathrm{~N} & -1.273614 & 0.573496 & 0.000000 \\ \mathrm{O} & -0.387596 & 3.239507 & 0.000000 \\ \mathrm{O} & 3.881533 & 1.804466 & 0.000000 \\ \mathrm{C} & 1.063829 & -0.101876 & 0.000000 \\ \mathrm{C} & -0.031003 & 0.883721 & 0.000000 \\ \mathrm{C} & 0.379884 & 2.320068 & 0.000000 \\ \mathrm{C} & 2.725656 & 1.496755 & 0.000000 \\ \mathrm{C} & -0.675690 & -1.766943 & 0.000000 \\ \mathrm{C} & -1.634987 & -0.771411 & 0.000000 \\ \mathrm{C} & -1.062461 & -3.117210 & 0.000000 \\ \mathrm{C} & -2.404645 & -3.432424 & 0.000000 \\ \mathrm{C} & -3.384030 & -2.419879 & 0.000000 \\ \mathrm{C} & -2.999225 & -1.111083 & 0.000000 \\ \mathrm{H} & -3.714628 & -0.310358 & 0.000000 \\ \mathrm{H} & -4.425471 & -2.681569 & 0.000000 \\ \mathrm{H} & -2.703586 & -4.464471 & 0.000000 \\ \mathrm{H} & -0.313253 & -3.888502 & 0.000000 \\ \mathrm{H} & 2.081843 & 3.429699 & 0.000000 \\ \mathrm{H} & 1.367328 & -2.091613 & 0.000000 \\ & & & \end{array}$

${ }^{1}\left(\pi, \pi^{*}\right)_{\min }$

$\begin{array}{lrrr} & & & \\ N & .314291 & 0.139157 & 0.000000 \\ \mathrm{~N} & 1.793152 & 2.452086 & 0.000000 \\ \mathrm{~N} & 0.623010 & -1.349119 & 0.000000 \\ \mathrm{~N} & -1.389676 & 0.585696 & 0.000000 \\ \mathrm{O} & -0.308462 & 3.278709 & 0.000000 \\ \mathrm{O} & 3.929723 & 1.711153 & 0.000000 \\ \mathrm{C} & 0.950503 & -0.040364 & 0.000000 \\ \mathrm{C} & -0.025557 & 0.920027 & 0.000000 \\ \mathrm{C} & 0.399594 & 2.307126 & 0.000000 \\ \mathrm{C} & 2.748976 & 1.460690 & 0.000000 \\ \mathrm{C} & -0.674559 & -1.746082 & 0.000000 \\ \mathrm{C} & -1.672925 & -0.704436 & 0.000000 \\ \mathrm{C} & -1.041168 & -3.115739 & 0.000000 \\ \mathrm{C} & -2.349523 & -3.450712 & 0.000000 \\ \mathrm{C} & -3.370348 & -2.437122 & 0.000000 \\ \mathrm{C} & -3.051929 & -1.131424 & 0.000000 \\ \mathrm{H} & -3.798427 & -0.360359 & 0.000000 \\ \mathrm{H} & -4.400308 & -2.742736 & 0.000000 \\ \mathrm{H} & -2.639089 & -4.484961 & 0.000000 \\ \mathrm{H} & -0.272987 & -3.868753 & 0.000000 \\ \mathrm{H} & 2.133609 & 3.389614 & 0.000000 \\ \mathrm{H} & 1.377052 & -2.005061 & 0.000000\end{array}$


Table SI1 (cont). Cartesian coordinates $(\AA)$ for the relevant structures of the isoalloxazine ring.

${ }^{3}\left(\pi, \pi^{*}\right)_{\min }$

$\begin{array}{lrrr}\mathrm{N} & 2.312043 & 0.169143 & 0.000000 \\ \mathrm{~N} & 1.775758 & 2.488690 & 0.000000 \\ \mathrm{~N} & 0.646183 & -1.395849 & 0.000000 \\ \mathrm{~N} & -1.358263 & 0.576828 & 0.000000 \\ \mathrm{O} & -0.355967 & 3.234728 & 0.000000 \\ \mathrm{O} & 3.910584 & 1.762289 & 0.000000 \\ \mathrm{C} & 1.010357 & -0.075074 & 0.000000 \\ \mathrm{C} & -0.002635 & 0.891606 & 0.000000 \\ \mathrm{C} & 0.399375 & 2.300506 & 0.000000 \\ \mathrm{C} & 2.738558 & 1.502350 & 0.000000 \\ \mathrm{C} & -0.674506 & -1.763672 & 0.000000 \\ \mathrm{C} & -1.653614 & -0.713304 & 0.000000 \\ \mathrm{C} & -1.062063 & -3.117522 & 0.000000 \\ \mathrm{C} & -2.379102 & -3.446568 & 0.000000 \\ \mathrm{C} & -3.375273 & -2.423105 & 0.000000 \\ \mathrm{C} & -3.032981 & -1.118084 & 0.000000 \\ \mathrm{H} & -3.768530 & -0.336472 & 0.000000 \\ \mathrm{H} & -4.412160 & -2.705779 & 0.000000 \\ \mathrm{H} & -2.677717 & -4.478064 & 0.000000 \\ \mathrm{H} & -0.302746 & -3.879837 & 0.000000 \\ \mathrm{H} & 2.101906 & 3.431370 & 0.000000 \\ \mathrm{H} & 1.375740 & -2.074079 & 0.000000\end{array}$

${ }^{3}\left(\mathbf{n}, \pi^{*}\right)_{\min }$

$\begin{array}{lrrr}\mathrm{N} & 2.303968 & 0.202840 & 0.000000 \\ \mathrm{~N} & 1.783250 & 2.508670 & 0.000000 \\ \mathrm{~N} & 0.633150 & -1.387680 & 0.000000 \\ \mathrm{~N} & -1.237485 & 0.504746 & 0.000000 \\ \mathrm{O} & -0.356709 & 3.263048 & 0.000000 \\ \mathrm{O} & 3.907791 & 1.799977 & 0.000000 \\ \mathrm{C} & 1.049235 & -0.079668 & 0.000000 \\ \mathrm{C} & 0.024520 & 0.936236 & 0.000000 \\ \mathrm{C} & 0.407125 & 2.333560 & 0.000000 \\ \mathrm{C} & 2.752211 & 1.528285 & 0.000000 \\ \mathrm{C} & -0.701379 & -1.782104 & 0.000000 \\ \mathrm{C} & -1.698637 & -0.791849 & 0.000000 \\ \mathrm{C} & -1.083344 & -3.120633 & 0.000000 \\ \mathrm{C} & -2.435190 & -3.456299 & 0.000000 \\ \mathrm{C} & -3.407651 & -2.467314 & 0.000000 \\ \mathrm{C} & -3.047805 & -1.134108 & 0.000000 \\ \mathrm{H} & -3.787308 & -0.355730 & 0.000000 \\ \mathrm{H} & -4.447770 & -2.735547 & 0.000000 \\ \mathrm{H} & -2.719770 & -4.492061 & 0.000000 \\ \mathrm{H} & -0.331286 & -3.889231 & 0.000000 \\ \mathrm{H} & 2.118518 & 3.448646 & 0.000000 \\ \mathrm{H} & 1.349458 & -2.078601 & 0.000000\end{array}$



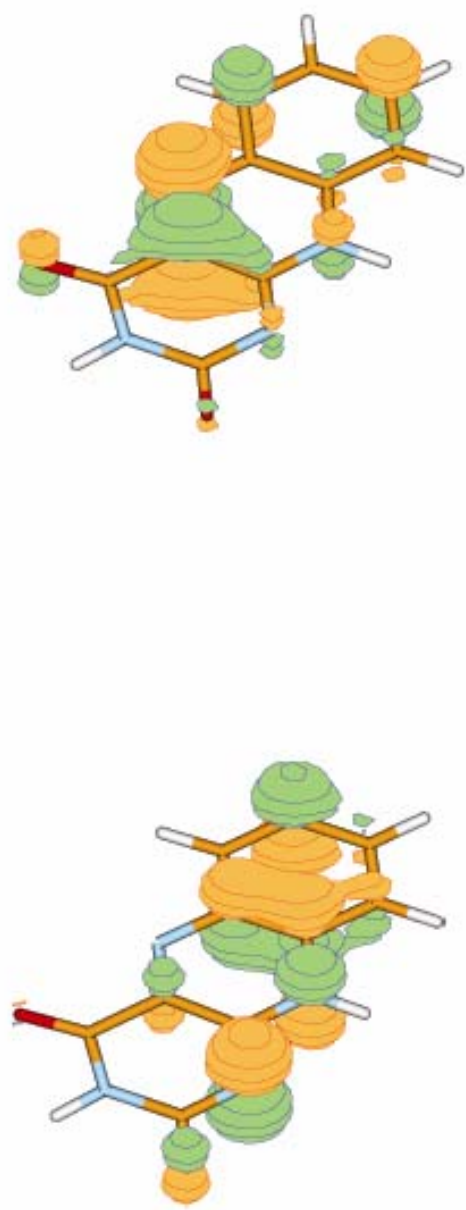

Figure SI1. LUMO-like (top) and HOMO-like (bottom) of the CASSCF $(14,13)$ natural orbitals. 


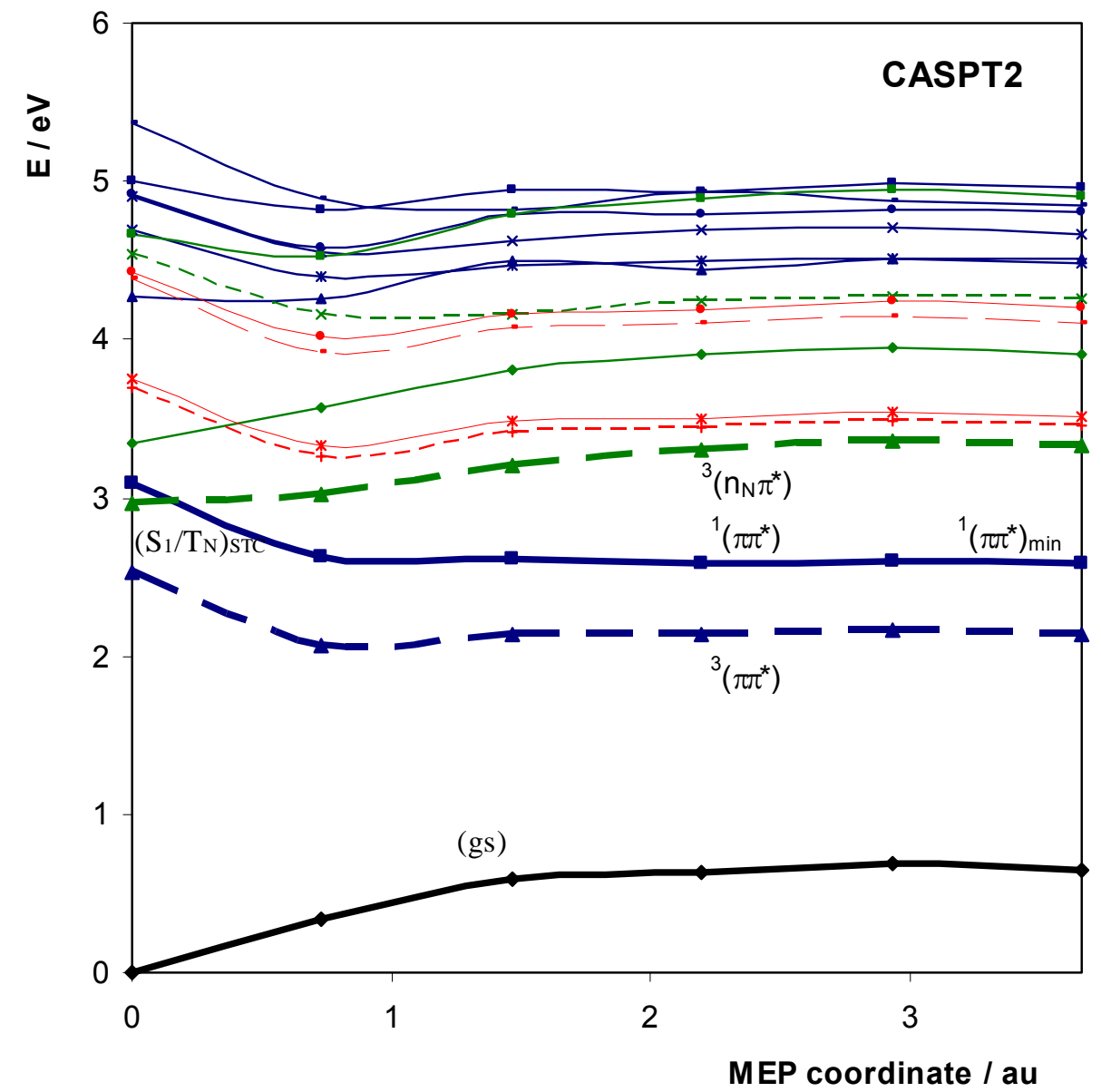

Figure SI2. CASPT2 evolution of the ground and excited states along the $\mathrm{S}_{1}$-MEP. 


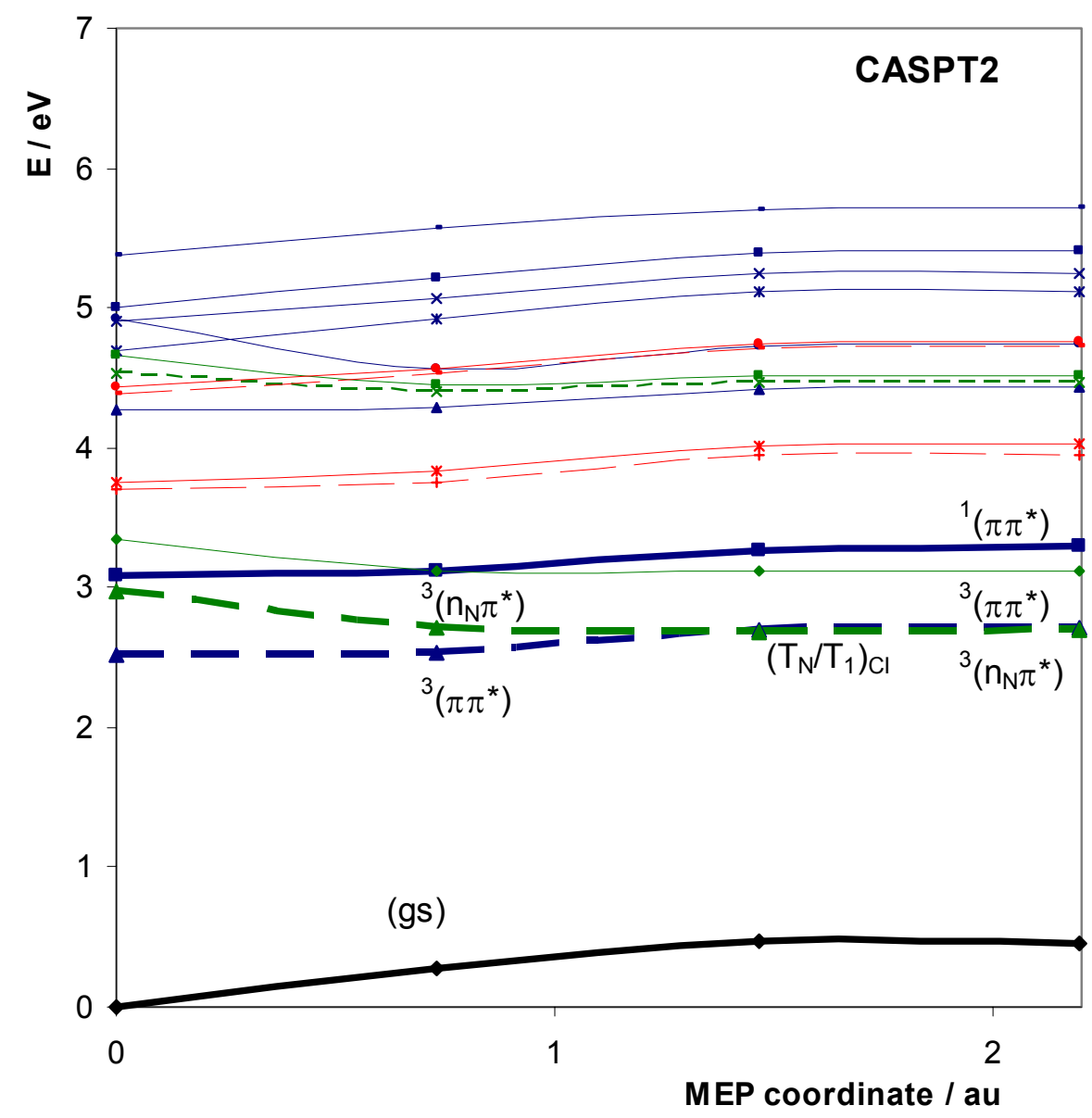

Figure SI3. CASPT2 evolution of the ground and excited states along the TN-MEP from $\left(\mathrm{S}_{1} / \mathrm{T}_{\mathrm{N}}\right) \mathrm{sTc}$. 


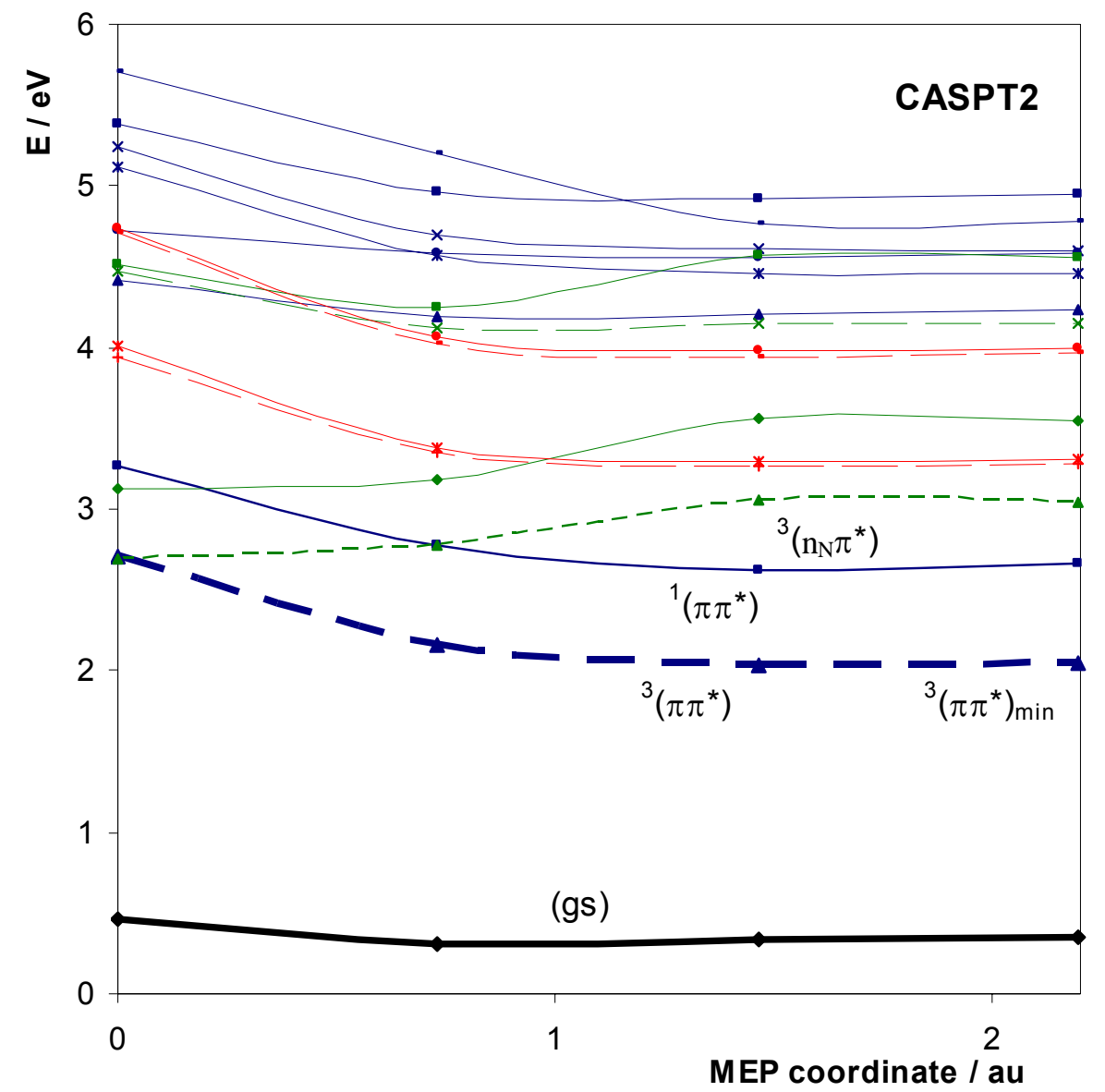

Figure SI4. CASPT2 evolution of the ground and excited states along the T1-MEP from $\left(\mathrm{T}_{\mathrm{N}} / \mathrm{T}_{1}\right)_{\mathrm{CI}}$ 